\title{
Composition of Path Transductions
}

\author{
Tanguy Urvoy \\ Université Paul Sabatier, IRIT, Batiment 1R1, \\ 118 route de Narbonne - 31062 Toulouse cedex 4 \\ urvoy@irit.fr \\ http://www.irit.fr/recherches/TYPES/SVF/urvoy/
}

\begin{abstract}
We propose to study two infinite graph transformations that we respectively call bounded and unbounded path transduction. These graph transformations are based on path substitutions and graph products. When graphs are considered as automata, path transductions correspond to rational word transductions on the accepted languages. They define strict subclasses of monadic transductions and preserve the decidability of monadic second order theory.

We give a generalization of the Elgot and Mezei composition theorem from rational word transductions to path transductions.
\end{abstract}

\section{Introduction}

As a theoretical model for systems verification, the study of infinite transitions graphs has become an active research area. The most fundamental dynamic behavior of these (oriented, labelled and simple) graphs is the language of their path labels: a class of automata corresponds to each class of graphs.

To be interesting for formal verification, an infinite graph is also expected to have some decidable properties like reachability or a decidable monadic second order theory. These decidability results for graphs are often strongly connected with the classes of languages recognized by the corresponding automata: graphs hierarchies share many properties with classical languages hierarchies [16].

A standard way to show a property on a given infinite structure is to define this structure by transformations from a known generator. If the transformations used are well suited, the property will be inherited from the generator. A transformation which preserve a given property is said to be compatible with this property.

For example, monadic transductions [7] and unfolding [8] are compatible with monadic second order logic: any graph which is built by these transformations from a finite graph will have a decidable monadic theory [5]. Monadic second order logic is very expressive and thus undecidable for many graphs. For these graphs one must resign oneself to weaker decidability results like reachability or reachability under rational control. To study such properties, one must consider graph transformations which are strictly weaker than monadic transductions.

This article is the continuation of [17] which extended to infinite graphs the classical language theoretic notion of abstract family [11,12]. Many families of 
graphs, like higher order pushdown graphs [5], Petri nets transitions graphs or automatic graphs [3] can be characterized by using a common and restricted set of simple graph transformations from specific generating systems [18].

Abstract families are a way to build generic proofs: we consider families of graphs without specifying what the graphs are. We only require that the families are closed under certain operations. In language theory, rational word transductions $[12,2]$ are one of these fundamental operations.

Rational transductions have been naturally extended from words to trees [15] but no convenient automaton based definition of graph transduction have been given. In this article we study two graph transformations that we respectively call bounded path transduction and unbounded path transduction. These transformations are not defined in terms of finite automaton nor in terms of logic but rather in terms of path substitution, path morphism and graph product. They define strict subclasses of monadic transductions [7] which preserve the decidability of monadic second order theory but their principal characteristic is to respect the orientation of edges and thus being compatible with language transformations.

Given a path transduction $T$, we can build a word rational transduction $\hat{T}$ such that for any automaton $\mathcal{G}$ (finite or not) we have:

$$
L(T(\mathcal{G}))=\hat{T}(L(\mathcal{G})) .
$$

Conversely, given a rational word transduction $R$, we easily build a path transduction $T$ verifying $R=\hat{T}$.

The most important and new result is the generalization of the Elgot and Mezei composition closure theorem from rational word transductions to path transductions.

This article is divided into four parts : the first part recalls some basic definitions about graphs, automata and rational word transductions; the second one introduces the notion of (language) compatible transformation and gives a formal definition of path transductions; the third one is devoted to bounded path transductions and the last to unbounded path transductions.

\section{Preliminaries}

\subsection{Graphs and Automata}

We use the standard definition of graphs and automata but here the state space is not supposed to be finite.

Definition 1. A $\Sigma$ - $\Gamma$-graph structure (or more simply a $\Sigma$-graph or a graph) is a tuple $\left\langle\Sigma, \Gamma, Q,\left(R_{a}\right)_{a \in \Sigma},\left(P_{b}\right)_{b \in \Gamma}\right\rangle$ where:

$-\Sigma$ is an alphabet of edge labels;

$-\Gamma$ is an alphabet of states labels;

- $Q$ is a countable set of states (or vertices);

- for each label $a \in \Sigma, R_{a} \subseteq Q \times Q$ is the relation labelled by a; 
- for each label $b \in \Gamma, P_{b} \subseteq Q$ is the unary predicate (the set) labelled by $b$;

Recall that the composition of two relations $R$ and $S$ on $Q$ is the relation

$$
R \cdot S=\{(p, q) \in Q \times Q \mid \exists r \in Q, p R r, r S q\} .
$$

The mapping which associates to each symbol $a \in \Sigma$ its relation $R_{a}$ is extended to words according to the following rules:

$$
\begin{aligned}
R_{\varepsilon} & =\{(p, p) \mid p \in Q\} & & (\varepsilon \text { denotes the empty word }) \\
R_{a u} & =R_{a} \cdot R_{u} & & a \in \Sigma, u \in \Sigma^{*} .
\end{aligned}
$$

We will also consider the relation labelled by a set $L$ of words:

$$
R_{L}=\left\{(p, q) \mid \exists w \in L, p R_{w} q\right\} .
$$

The path label language of $\mathcal{G}$ between two sets of vertices $A$ and $B$ is the set of finite words

$$
L(\mathcal{G}, A, B)=\left\{w \in \Sigma^{*} \mid R_{w} \cap A \times B \neq \emptyset\right\} .
$$

An automaton is a graph structure with two unary predicates : $P_{i}$ for the initial states and $P_{f}$ for the final ones. The language $L(\mathcal{A})$ of an automaton is the path label language $L\left(\mathcal{A}, P_{i}, P_{f}\right)$.

\subsection{Word Transductions}

Rational word transductions are fundamental tools for the study of formal languages. We give here some basic definitions of this concept. See $[1,2,12]$ for further details.

Definition 2. A rational transduction between $\Sigma_{1}^{*}$ and $\Sigma_{2}^{*}$ is a rational subset of the monoid $\Sigma_{1}^{*} \times \Sigma_{2}^{*}$ for the canonical concatenation $(u, v) \cdot(w, x)=(u w, v x)$.

A rational transduction is usually defined, as in Figure 1, by a finite automaton, called a transducer, which is labelled by pairs of words. An equivalent way to define such a relation is to give its morphism decomposition:

Property 1. Any rational transduction $R$ is the composition of an inverse morphism $f^{-1}$, an intersection with a rational language $K$, and a direct morphism $g$ :

$$
R=f^{-1} \cdot(\cap K) \cdot g=\{(f(w), g(w)) \mid w \in K\} .
$$

A relation $R$ between $\Sigma_{1}^{*}$ and $\Sigma_{2}^{*}$ is faithful when for any $w \in \Sigma_{2}^{*}, R^{-1}(w)$ is finite; it is continuous when $R^{-1}(\{\varepsilon\})=\{\varepsilon\}$. These two properties are important for the study of quasi-real-time acceptors [10] because a transduction which is both faithful and continuous is unable to erase more than a bounded number of letters.

We give here a representation of faithful and continuous rational transductions which was given in [4]. Recall that a morphism $\alpha$ from $\Sigma_{1}^{*}$ to $\Sigma_{2}^{*}$ is strictly alphabetic when $\alpha(a) \in \Sigma_{2}$ for any $a \in \Sigma_{1}$. 


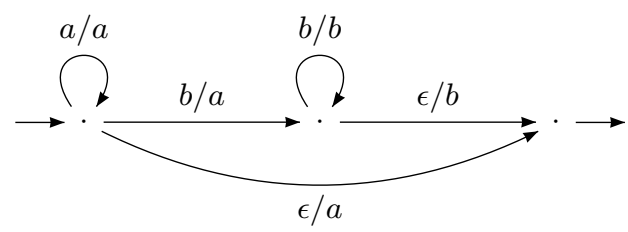

Fig. 1. A transducer recognizing the function $a^{n} b^{m} \mapsto a^{n+1} b^{m}$.

Theorem 1. (Boasson and Nivat) A rational transduction $R$ between $\Sigma^{*}$ and $\Sigma_{2}^{*}$ is faithful and continuous if and only if there exists an alphabet $\Pi$, a rational set $K \in \operatorname{Rat}\left(\Pi^{*}\right)$, a morphism $f$ from $\Pi^{*}$ to $\Sigma^{*}$ and a strictly alphabetic morphism $\alpha$ from $\Pi^{*}$ to $\Sigma_{2}^{*}$ such that

$$
R=\{(f(w), \alpha(w)) \mid w \in K\} .
$$

The Elgot and Mezei composition theorem is an important property of rational transductions.

Theorem 2. (Elgot and Mezei)

The rational transductions are closed under composition.

A proof of this result can be found in [1] or [2].

Because faithfulness and continuity are preserved by composition we have the following corollary.

Corollary 1. Faithful and continuous rational transductions are closed by composition.

Rational transductions and their closure by composition are fundamentals in the theory of abstract families of languages (AFL). Figure 2 gives some of the most usual closure operators and the names of the corresponding abstract families. See [12,2] or [10] for further details on this theory.

\section{Language Compatible Graph Transformations : Path Transductions}

We study graph transformations which are independent of the vertices naming convention or, in other words, invariants with respect to graph isomorphism. Another fundamental restriction is that we only consider graph transformations that are "compatible" with language transformations.

Definition 3. A graph transformation $T$ of arity $n$ is compatible (with language transformations) if there exists a language transformation $\hat{T}$ of arity $n$ such that for any sequence $\left(\mathcal{A}_{i}\right)_{1 \leq i \leq n}$ of automata, we have

$$
L\left(T\left(\mathcal{A}_{1}, \ldots, \mathcal{A}_{n}\right)\right)=\hat{T}\left(L\left(\mathcal{A}_{1}\right), \ldots, L\left(\mathcal{A}_{n}\right)\right) .
$$




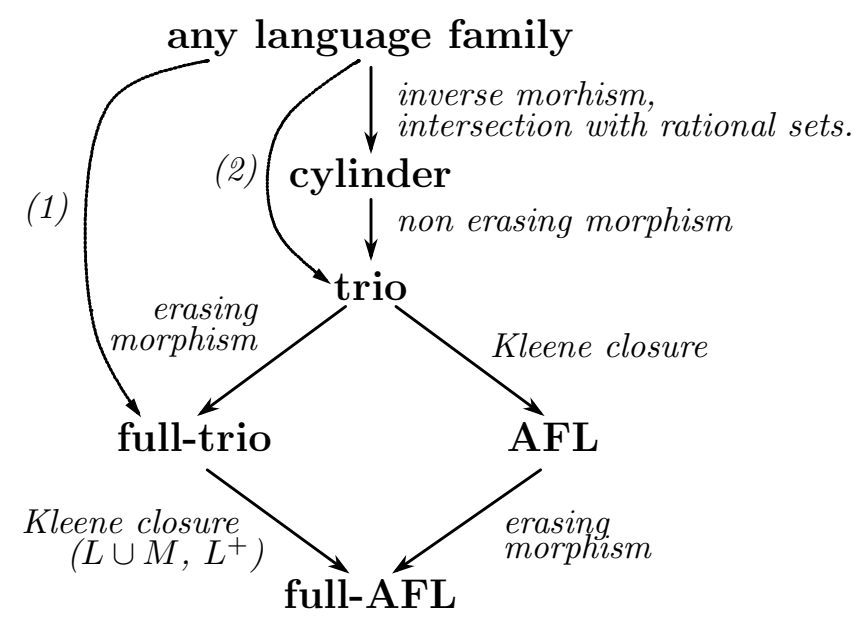

Fig. 2. Different abstract families of languages from the less constrained to the most constrained. (1) stands for rational transductions and (2) for faithful and continuous rational transductions.

Many graph transformations are impossible to translate into language transformations. Consider, for example, the simple transformation $B$ consisting of adding a backward edge labelled by $\bar{a}$ for each edge labelled by an $a$. The non compatibility of this transformation is illustrated by Figure 3 .

\subsection{Path Morphisms and Path Substitutions}

A morphism is a mapping which replace letters by words. More generally, a substitution is a relation which replaces letters by languages. Morphisms, inverse morphism, substitutions and inverse substitutions are well-known language transformations but these transformations can be generalized as graph/automata transformations.

Definition 4. Let $\Sigma_{1}$ and $\Sigma_{2}$ be alphabets and $h \subseteq \Sigma_{1} \times \Sigma_{2}^{*}$ be a relation.

The substitution generated by $h$ is the relation $\bigcup_{n \geq 0} h_{n}$ defined inductively by:

$$
\begin{aligned}
h_{0} & =\{(\varepsilon, \varepsilon)\} \\
h_{n+1} & =\left\{(a u, v w) \in \Sigma_{1}^{n+1} \times \Sigma_{2}^{*} \mid a \in \Sigma_{1}, v \in h(a), w \in h_{n}(u)\right\} .
\end{aligned}
$$

We use the same symbol to denote the substitution $\bigcup_{n \geq 0} h_{n}$ and its generator $h$.

Example 1. If $h$ is the rational substitution defined by $h(c)=a$ and $h(d)=(a b)^{*}$, the image of the word $c d c d$ by $h$ is the rational language $a(a b)^{*} a(a b)^{*}$. By inverse we have $h^{-1}(a b a b)=\{w \mid a b a b \in h(w)\}$ hence $h^{-1}(a b a b)=d^{+}$.

When the relation is a function, which associates a unique word to each letter, the generated substitution is a morphism, when this image is a rational (resp. finite) language, the substitution is called rational (resp. finite). 


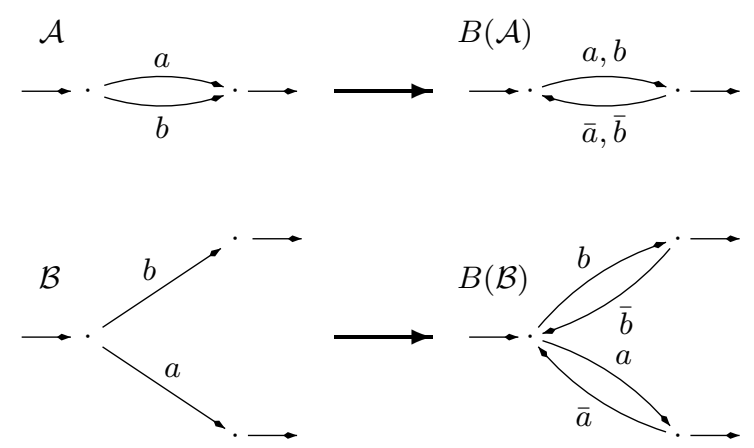

Fig. 3. The two finite automata $\mathcal{A}$ and $\mathcal{B}$ are both accepting the language $\{a, b\}$, but their respective images by $B$ do not accept the same languages.

Inverse rational substitution is a simple and well defined operation on graphs. Each path labelled by a word in the language $h(a)$ is replaced by an arc labelled by $a$. This path transformation do not add vertices to the graph.

Definition 5. (Caucal [6])

If $h$ is a substitution from $\Sigma_{1}^{*}$ to $\Sigma_{2}^{*}$ and $\mathcal{G}$ is a graph, then $h^{-1}(\mathcal{G})$ is the graph $\left\langle\Sigma_{1}, \Gamma^{\mathcal{G}}, Q^{\mathcal{G}},\left(R_{a}^{h^{-1}(\mathcal{G})}\right)_{a \in \Sigma_{1}},\left(P_{b}\right)_{b \in \Gamma^{\mathcal{G}}}\right\rangle$ where

$$
R_{a}^{h^{-1}(\mathcal{G})}=R_{h(a)}^{\mathcal{G}} \text { for each } a \in \Sigma_{1}
$$

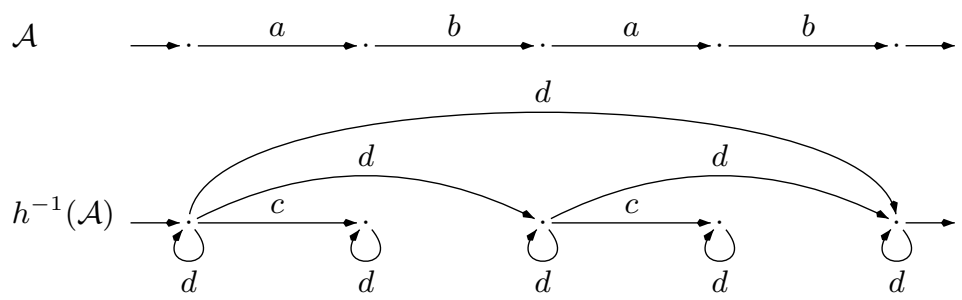

Fig. 4. An automaton $\mathcal{A}$ and its inverse rational substitution according to $h(c)=a$ and $h(d)=(a b)^{*}$. In other words $R_{c}^{h^{-1}(\mathcal{A})}=R_{a}^{\mathcal{A}}$ and $R_{d}^{h^{-1}(\mathcal{A})}=\left(R_{a}^{\mathcal{A}} \cdot R_{b}^{\mathcal{A}}\right)^{*}$.

From the algebraic point of view this transformation is natural: the set of relations $\left\{R_{w}^{\mathcal{G}} \mid w \in \Sigma_{2}^{*}\right\}$ is a monoid for the composition operator. When $h$ is rational (resp. finite), each relation $R_{a}^{h^{-1}(\mathcal{G})}$ is a rational (resp. finite) subset of 
this monoid. Figure 4 gives an example of inverse rational substitution. Note that the languages $L\left(h^{-1}(\mathcal{A})\right)$ and $h^{-1}(L(\mathcal{A}))$ coincide: inverse substitution is a compatible transformation.

Lemma 1. Let $\mathcal{G}=\left\langle\Sigma_{1}, \Gamma, Q,\left(R_{a}\right)_{a \in \Sigma_{1}},\left(P_{b}\right)_{b \in \Gamma}\right\rangle$ be a graph, let $i, f$ be two letters of $\Gamma$ and let $g, h$ be substitutions between $\Sigma_{2}^{*}$ and $\Sigma_{1}^{*}$.

1. $\forall w \in \Sigma_{2}^{*}, R_{w}^{h^{-1}(\mathcal{G})}=R_{h(w)}^{\mathcal{G}}$;

2. $L\left(h^{-1}(\mathcal{G}), P_{i}, P_{f}\right)=h^{-1}\left(L\left(\mathcal{G}, P_{i}, P_{f}\right)\right)$;

3. $g^{-1}\left(h^{-1}(\mathcal{G})\right)=(g \cdot h)^{-1}(\mathcal{G})$ (relational composition.).

Recall that a monoid morphism $\alpha$ from $\Sigma_{1}^{*}$ to $\Sigma_{2}^{*}$ is strictly alphabetic when $\alpha(a) \in \Sigma_{2}$ for any $a \in \Sigma_{1}$. If $\alpha$ is strictly alphabetic, we write $\alpha(\mathcal{G})$ for the graph obtained by replacing each $R_{a}$ by $R_{\alpha(a)}$. Direct strictly alphabetic mapping is, like inverse morphism, a special case of inverse finite substitution.

It is harder to give for graphs a definition of direct rational substitution that extend cleanly the language transformation. The most natural way, given a continuous ${ }^{1}$ substitution $h$, is to use an edge replacement [9] to replace each edge of the graph labelled by a symbol $a$ by the automaton $\mathcal{G}_{a}$ recognizing the language $h(a)$.

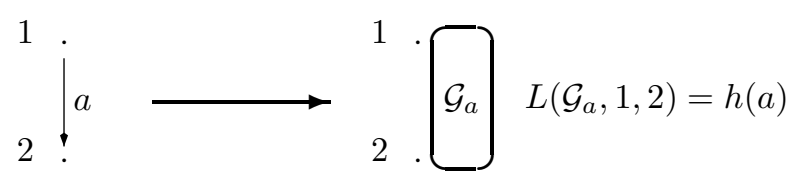

As proved in [17], such an (oriented) edge replacement may be simulated by using only finite inverse substitution and product.

\subsection{Graph Products}

The notion of graph product is well known in automata theory for its correspondence with the intersection of languages.

Definition 6. Let $\mathcal{G}$ and $\mathcal{H}$ be two graphs. The product $\mathcal{G} \times \mathcal{H}$ is the graph

$$
\left\langle\Sigma^{\mathcal{G}} \cap \Sigma^{\mathcal{H}}, Q^{\mathcal{G}} \times Q^{\mathcal{H}},\left(R_{a}\right)_{a \in \Sigma^{\mathcal{G}} \cap \Sigma^{\mathcal{H}}},\left(P_{b}\right)_{b \in \Gamma^{\mathcal{G}} \cap \Gamma^{\mathcal{H}}}\right\rangle
$$

where

$$
\begin{aligned}
R_{a} & =\left\{\left(\left(p, p^{\prime}\right),\left(q, q^{\prime}\right)\right) \mid p R_{a}^{\mathcal{G}} q, p^{\prime} R_{a}^{\mathcal{H}} q^{\prime}\right\} \quad \text { for each } a \in \Sigma^{\mathcal{G}} \cap \Sigma^{\mathcal{H}} \text { and } \\
P_{b} & =P_{b}^{\mathcal{G}} \times P_{b}^{\mathcal{H}} \text { for each } b \in \Gamma^{\mathcal{G}} \cap \Gamma^{\mathcal{H}} .
\end{aligned}
$$

Lemma 2. Let $\mathcal{G}$ and $\mathcal{H}$ be two graphs.

\footnotetext{
${ }^{1}$ if $\varepsilon \in h(a)$ we need $\varepsilon$-transitions or vertex fusion.
} 
1. For any word $w$, we have:

$$
R_{w}^{\mathcal{G} \times \mathcal{H}}=\left\{\left(\left(p, p^{\prime}\right),\left(q, q^{\prime}\right)\right) \mid p R_{w}^{\mathcal{G}} q, p^{\prime} R_{w}^{\mathcal{H}} q^{\prime}\right\} ;
$$

2. for any couple of letters $i, f$ :

$$
L\left(\mathcal{G} \times \mathcal{H}, P_{i}^{\mathcal{G}} \times P_{i}^{\mathcal{H}}, P_{f}^{\mathcal{G}} \times P_{f}^{\mathcal{H}}\right)=L\left(\mathcal{G}, P_{i}^{\mathcal{G}}, P_{f}^{\mathcal{G}}\right) \cap L\left(\mathcal{H}, P_{i}^{\mathcal{H}}, P_{f}^{\mathcal{H}}\right) ;
$$

3. For any morphism $f$, we have $f^{-1}(\mathcal{G} \times \mathcal{H})=f^{-1}(\mathcal{G}) \times f^{-1}(\mathcal{H})$.

\subsection{Definition of Path Transductions}

In [17], an abstract family of graphs $(A F G)$ is defined as a graph family closed by inverse finite substitution and product by finite graphs. A full- $A F G$ is an $A F G$ which is also closed by inverse rational substitution. These two definitions only ask the considered family to be closed by these operations but a question was still remaining for principal $A F G$ (resp. principal full- $A F G$ ). An $A F G$ (respectively a full- $A F G$ ) is principal when all its elements are derived from a single graph (the generator) by a finite sequence of $A F G$ (resp. full- $A F G$ ) operations.

Does the AFG transformations generate strictly increasing (for inclusion) chains of graph families? If not, how many successive transformations are needed to obtain the whole family from its generator?

The answer to the second question is three and the transformations obtained by composition of the three basic AFG transformations used are, as proved in the next sections, what we call path transductions. We distinguish two classes of path transductions : bounded path transduction which are only local transformations and unbounded path transduction which may act more globally on the graph.

\section{Definition 7.}

1. A bounded path transduction is the composition of an inverse morphism, a product by a finite graph and a direct strictly alphabetic morphism;

2. an unbounded path transduction is the composition of an inverse rational substitution, a product by a finite graph and an inverse rational substitution.

It is important to notice that the definition of bounded path transductions is a direct generalization to graphs of Boasson Nivat's characterization of faithful and continuous rational word transductions (Theorem 1). The only difference is that instead of using word morphisms, we use path morphisms and instead of using intersection with rational sets we use product with finite graphs.

The characterization of general rational word transductions with inverse substitution is less usual but easy to deduce from Property 1.

Property 2. Any rational word transduction is the composition of an inverse rational word substitution, an intersection by a rational set and an inverse rational substitution.

Theorem 3. 1. Given a word transduction $t$ we can construct an unbounded path transduction $T$ such that $\hat{T}=t$. 
2. given a faithful and continuous word transduction $t$ we can construct a bounded path transduction $T$ such that $\hat{T}=t$.

\section{Proof:}

The main proof is already given in [17]. For (2) we can use theorem 1.

\subsection{Examples}

Here is the example of a bounded transduction $T$, defined by a morphism $f$, a finite automaton $\mathcal{H}$ and a strictly alphabetic morphism $\alpha$.

$$
f:\{\begin{array}{l}
A \mapsto a \\
B \mapsto \bar{a} \\
C \mapsto a a \\
D \mapsto \varepsilon \\
E \mapsto \bar{a}
\end{array}, \quad \mathcal{H}: \overbrace{B}^{A} D \text {, and } \alpha:\left\{\begin{array}{l}
A \mapsto a \\
B \mapsto \bar{a} \\
C \mapsto b \\
D \mapsto c \\
E \mapsto d
\end{array}\right.
$$

This path transduction may also be represented directly, as in figure 5 , by a finite transducer. The application of the path transduction is a kind of "left synchronized graph product".

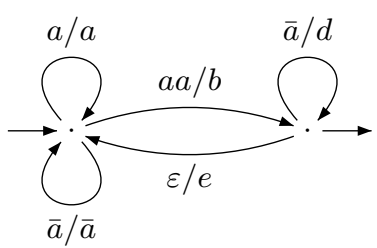

Fig. 5. Another representation of $T$.

The one letter Dyck graph, illustrated on Figure 6 is a representation of the positive integers with +1 and -1 operations respectively encoded by the labels $a$ and $\bar{a}$. This graph is a generator for the one counter automata transition graphs.

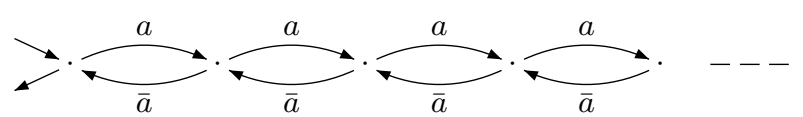

Fig. 6. The one letter Dyck graph $\Delta_{1}$. 


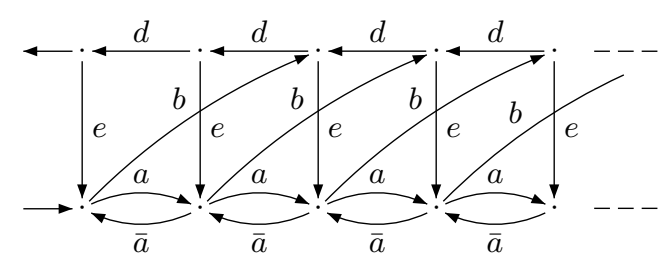

Fig. 7. The graph $T\left(\Delta_{1}\right)$.

By applying $T$ to $\Delta_{1}$, we obtain the graph of figure 7 .

Figure 8, give an example of unbounded path transduction and its application to $\Delta_{1}$. The $\stackrel{\bar{a}^{*} / c}{\rightarrow}$ edge of the transducer leads to infinite degrees in the final graph.

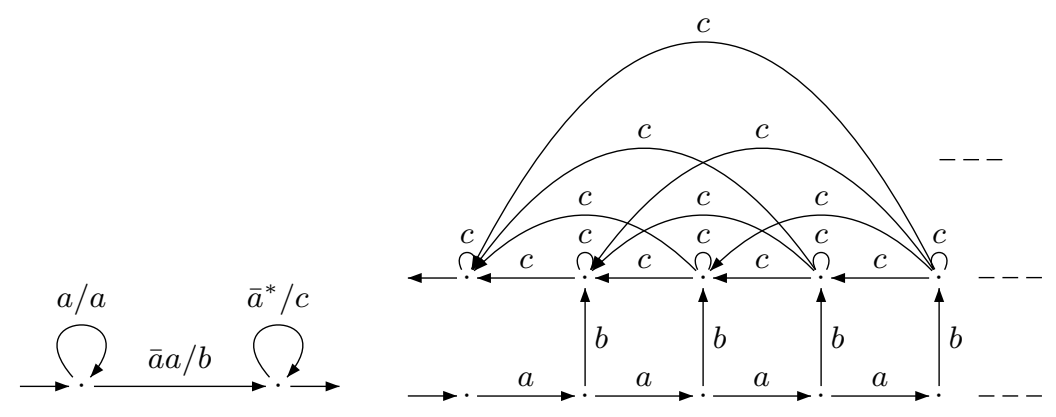

Fig. 8. An unbounded path transduction and its application to $\Delta_{1}$.

The two letters Dyck graph, illustrated on Figure 9 is a binary tree with backward transitions. Its path label language from and to the "root" is the semiDyck language a generator of context-free languages but its "geometry" is also generating, by path transductions, the pushdown graphs and prefix recognizable graphs which are the prefix transitions graphs of finite (resp. recognizable) word rewriting systems ${ }^{2}[13,6]$.

We can deduce the decidability of monadic second order theory for these graphs from the fundamental result of Rabin [14] about the binary tree. More examples like Petri nets transitions graphs or automatic graphs can be found in [18].

\footnotetext{
${ }^{2}$ The definition of [13] use a restriction to a reachable component and the one of [6] use a restriction to rational sets of vertices. We consider here the prefix rewriting graphs without these restrictions.
} 


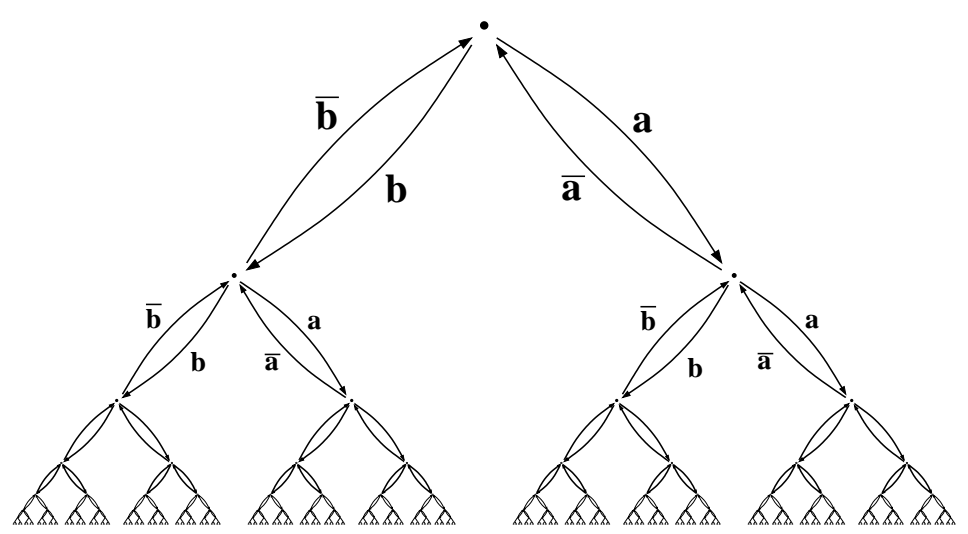

Fig. 9. A generator of pushdown AFG and prefix recognizable full-AFG. This graph has a decidable second order monadic theory [14].

\section{Composition of Bounded Path Transductions}

In this section we show that the classical Elgot and Mezei theorem naturally extends from faithful and continuous rational word transductions to bounded path transductions.

To establish this result, we use the basic properties of inverse morphism and product (Lemma 1 and Lemma 2) but the keys of the proof are the two following "delayed forgetting" lemmas.

Lemma 3. If $f$ is a morphism and $\alpha$ is a strictly alphabetic morphism, then we can construct a morphism $g$ and a strictly alphabetic morphism $\beta$ such that for any graph $\mathcal{G}$, we have

$$
f^{-1}(\alpha(\mathcal{G}))=\beta\left(g^{-1}(\mathcal{G})\right)
$$

Proof: We first remark that the relational composition $h=f \cdot \alpha^{-1}$, which verifies $h^{-1}(\mathcal{G})=f^{-1}(\alpha(\mathcal{G}))$, is a finite substitution. For each letter $a \in \operatorname{Dom}(h)$, we define the (finite) alphabet $\Sigma_{a}=\{[a, w] \mid w \in h(a)\}$ and take $\beta([a, w])=a$ and $g([a, w])=w$ to get $g\left(\beta^{-1}(a)\right)=h(a)$

In other words, the direct strictly alphabetic morphism, which forgets informations, may be applied after the inverse morphism.

Lemma 4. If $\mathcal{H}$ is a graph and $\alpha$ is a strictly alphabetic morphism, then for any graph $\mathcal{G}$, we have

$$
\alpha(\mathcal{G}) \times \mathcal{H}=\alpha\left(\mathcal{G} \times \alpha^{-1}(\mathcal{H})\right)
$$


Proof: Let $a$ be a label of $\mathcal{H}$.

$$
\begin{aligned}
R_{a}^{\alpha(\mathcal{G}) \times \mathcal{H}} & =\left\{\left(\left(p, p^{\prime}\right),\left(q, q^{\prime}\right)\right) \mid p R_{a}^{\alpha(\mathcal{G})} q, p^{\prime} R_{a}^{\mathcal{H}} q^{\prime}\right\} \quad \text { (by product def.) } \\
& =\left\{\left(\left(p, p^{\prime}\right),\left(q, q^{\prime}\right)\right) \mid \exists b, a=\alpha(b), p R_{b}^{\mathcal{G}} q, p^{\prime} R_{a}^{\mathcal{H}} q^{\prime}\right\} \quad \text { (by } \alpha(\mathcal{G}) \text { def.) } \\
& =\left\{\left(\left(p, p^{\prime}\right),\left(q, q^{\prime}\right)\right) \mid \exists b, a=\alpha(b), p R_{b}^{\mathcal{G}} q, \exists c, c=\alpha(b), p^{\prime} R_{c}^{\alpha^{-1}(\mathcal{H})} q^{\prime}\right\} \\
& \left.=\left\{\left(\left(p, p^{\prime}\right),\left(q, q^{\prime}\right)\right) \mid \exists b, a=\alpha(b), p R_{b}^{\mathcal{G}} q, p^{\prime} R_{a}^{\alpha^{-1}(\mathcal{H})} q^{\prime}\right\} \quad \text { (because } c=a\right) \\
& =R_{a}^{\alpha\left(\mathcal{G} \times \alpha^{-1}(\mathcal{H})\right)}
\end{aligned}
$$

Theorem 4. Bounded path transductions are closed by composition.

Proof: Let $T_{1}$ and $T_{2}$ be two bounded path transductions. Our aim is to show that the relation $T=T_{1} \cdot T_{2}$ remains a bounded path transduction. Let $\mathcal{G}$ be a graph and let $\mathcal{H}=T_{2}\left(T_{1}(\mathcal{G})\right)$ hence

$$
\mathcal{H}=\alpha_{2}\left(f_{2}^{-1}\left(\alpha_{1}\left(f_{1}^{-1}(\mathcal{G}) \times \mathcal{F}_{1}\right)\right) \times \mathcal{F}_{2}\right)
$$

where $f_{i}$ are morphisms, $\mathcal{F}_{i}$ are finite graphs and $\alpha_{i}$ are strictly alphabetic morphisms. By Lemma 3, there exists a morphism $g$ and a strictly alphabetic morphism $\beta$ such that $\alpha_{1} \cdot f_{2}^{-1}=g^{-1} \cdot \beta$, hence

$$
\mathcal{H}=\alpha_{2}\left(\beta\left(g^{-1}\left(f_{1}^{-1}(\mathcal{G}) \times \mathcal{F}_{1}\right)\right) \times \mathcal{F}_{2}\right) .
$$

By Lemma $2, g$ being a morphism, we have:

$$
g^{-1}\left(f_{1}^{-1}(\mathcal{G}) \times \mathcal{F}_{1}\right)=g^{-1}\left(f_{1}^{-1}(\mathcal{G})\right) \times g^{-1}\left(\mathcal{F}_{1}\right),
$$

hence

$$
\mathcal{H}=\alpha_{2}\left(\beta\left(g^{-1}\left(f_{1}^{-1}(\mathcal{G})\right) \times g^{-1}\left(\mathcal{F}_{1}\right)\right) \times \mathcal{F}_{2}\right) .
$$

By Lemma 1 , the composition of the two inverse morphisms $f_{1}^{-1}$ and $g^{-1}$ is an inverse morphism: if we set $h=g \cdot f_{1}$ and $\mathcal{F}=g^{-1}\left(\mathcal{F}_{1}\right)$, then we have:

$$
\mathcal{H}=\alpha_{2}\left(\beta\left(h^{-1}(\mathcal{G}) \times \mathcal{F}\right) \times \mathcal{F}_{2}\right) .
$$

By Lemma 4, we may substitute $\beta\left(h^{-1}(\mathcal{G}) \times \mathcal{F}\right) \times \mathcal{F}_{2}$ by $\beta\left(h^{-1}(\mathcal{G}) \times \mathcal{F} \times \beta^{-1}\left(\mathcal{F}_{2}\right)\right)$. With $\mathcal{K}=\mathcal{F} \times \beta^{-1}\left(\mathcal{F}_{2}\right)$ and $\gamma=\alpha_{2} \circ \beta$ :

for any graph $\mathcal{G}$

$$
\alpha_{2}\left(f_{2}^{-1}\left(\alpha_{1}\left(f_{1}^{-1}(\mathcal{G}) \times \mathcal{F}_{1}\right)\right) \times \mathcal{F}_{2}\right)=\gamma\left(h^{-1}(\mathcal{G}) \times \mathcal{K}\right)
$$

\section{Composition of Unbounded Path Transductions}

In this section we show that the classical Elgot and Mezei theorem naturally extends from rational word transductions to unbounded path transductions. The construction is similar as the one of Section 3 but, unlike inverse morphism, inverse rational substitution is not compatible with graph product. In general situations we have

$$
h^{-1}(\mathcal{G} \times \mathcal{H}) \neq h^{-1}(\mathcal{G}) \times h^{-1}(\mathcal{H}) .
$$


So we need a stronger "delay" property than Lemma 4 . This property is true up to isolated vertices.

A vertex/state is isolated if it is not connected to an edge nor labelled by a predicate. More formally recall that the image of a relation $R \subseteq Q \times Q$ by a function $f$ of domain $Q$ is $f(R)=\{(f(p), f(q)) \mid p R q\}$. We say that two $\Sigma-\Gamma$ graphs $\mathcal{G}$ and $\mathcal{H}$ are isomorphic up to isolated vertices, and we write $\mathcal{G} \approx \mathcal{H}$, if there is an injective function $f$ from $Q^{\mathcal{G}}$ to $Q^{\mathcal{H}}$ such that

$$
\forall a \in \Sigma, f\left(R_{a}^{\mathcal{G}}\right)=R_{a}^{\mathcal{H}} \text { and }, \forall b \in \Gamma, f\left(P_{b}^{\mathcal{G}}\right)=P_{b}^{\mathcal{H}} .
$$

Lemma 5. If $\mathcal{H}$ is a finite graph and $h$ is a rational word substitution then there exist a rational substitution $k$, a morphism $f$ and a finite graph $\mathcal{K}$ such that for any graph $\mathcal{G}$, we have

$$
h^{-1}(\mathcal{G}) \times \mathcal{H} \approx k^{-1}\left(f^{-1}(\mathcal{G}) \times \mathcal{K}\right) .
$$

Proof: For any letter $a$ appearing in $\operatorname{ran}(h)^{3}$, we define a fresh symbol $\tau_{a}$ (used to denote an $\varepsilon$-transition). We define the alphabetic morphism $f$, by

$$
\left\{\begin{array}{r}
f(a)=a \\
f\left(\tau_{a}\right)=\varepsilon
\end{array} \text { for any letter } a \text { appearing in } \operatorname{ran}(h) .\right.
$$

We build the graph $\mathcal{K}$ from the graph $\mathcal{H}$ by edge replacement: each edge labelled by $a$ is replaced by an automaton accepting the rational language $\tau_{a} h(a) \tau_{a}$.

When $\varepsilon \notin h(a)$ :

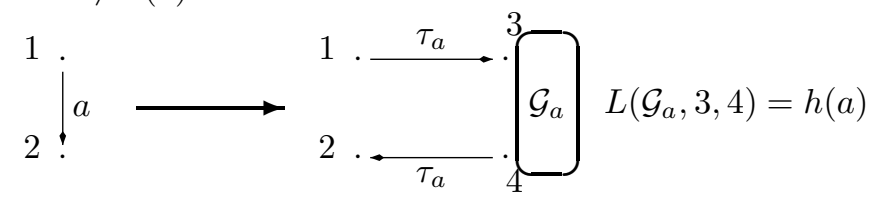

When $\varepsilon \in h(a)$ :

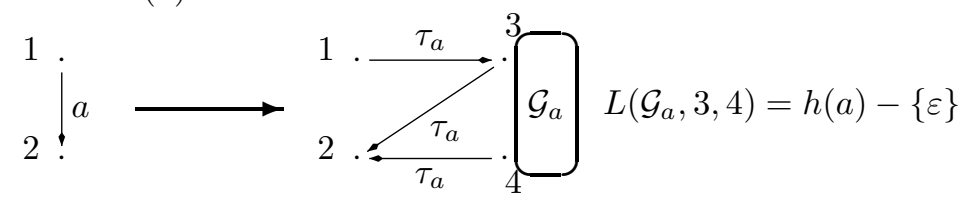

In other words, we have a direct substitution $\mathcal{K}=k(\mathcal{H})$ with $k$ defined by $k(a)=\tau_{a} h(a) \tau_{a}$ for all $a \in \operatorname{ran}(h)$ ( $\mathrm{k}$ is continuous). Note that for any couple of states $p, q \in Q^{\mathcal{K}}$, any label $a$ and any word $u \in h(a)$, we have

$$
p R_{a}^{\mathcal{H}} q \Leftrightarrow p R_{\tau_{a} u \tau_{a}}^{\mathcal{K}} q
$$

and

$$
p R_{u}^{\mathcal{G}} q \Leftrightarrow p R_{\tau_{a} u \tau_{a}}^{f^{-1}(\mathcal{G})} q
$$

\footnotetext{
$\overline{{ }^{3} \text { Recall that }} \operatorname{ran}(h)=\{v \mid \exists u, v \in h(u)\}$.
} 
Let $a$ be a label of $\mathcal{H}$ :

$$
\begin{aligned}
& R_{a}^{h^{-1}(\mathcal{G}) \times \mathcal{H}}=\left\{\left(\left(p, p^{\prime}\right),\left(q, q^{\prime}\right)\right) \mid \exists u \in h(a), p R_{u}^{\mathcal{G}} q, p^{\prime} R_{a}^{\mathcal{H}} q^{\prime}\right\} \\
& =\left\{\left(\left(p, p^{\prime}\right),\left(q, q^{\prime}\right)\right) \mid \exists u \in h(a), p R_{u}^{\mathcal{G}} q, p^{\prime} R_{\tau_{a} u \tau_{a}}^{\mathcal{K}} q^{\prime}\right\} \\
& =\left\{\left(\left(p, p^{\prime}\right),\left(q, q^{\prime}\right)\right) \mid \exists u \in h(a), p R_{\tau_{a} u \tau_{a}}^{f^{-1}(\mathcal{G})} q, p^{\prime} R_{\tau_{a} u \tau_{a}}^{\mathcal{K}} q^{\prime}\right\} \text { by (3) } \\
& =\left\{\left(\left(p, p^{\prime}\right),\left(q, q^{\prime}\right)\right) \mid \exists u \in h(a),\left(p, p^{\prime}\right) R_{\tau_{a} u \tau_{a}}^{f^{-1}(\mathcal{G}) \times \mathcal{K}}\left(q, q^{\prime}\right)\right\} \text { (Lemma 2) } \\
& =\left\{\left(\left(p, p^{\prime}\right),\left(q, q^{\prime}\right)\right) \mid \exists v \in k(a),\left(p, p^{\prime}\right) R_{v}^{f^{-1}(\mathcal{G}) \times \mathcal{K}}\left(q, q^{\prime}\right)\right\} k(a)=\tau_{a} h(a) \tau_{a}
\end{aligned}
$$

Hence, for any $a \in \operatorname{ran}(h)$, we have

$$
R_{a}^{h^{-1}(\mathcal{G}) \times \mathcal{H}}=R_{a}^{k^{-1}\left(f^{-1}(\mathcal{G}) \times \mathcal{K}\right)}
$$

The two graphs are isomorphic up to the vertices of $Q^{\mathcal{K}}-Q^{\mathcal{H}}$ which are isolated in $k^{-1}\left(f^{-1}(\mathcal{G}) \times \mathcal{K}\right)$.

Theorem 5. Up to isolated vertices, unbounded path transductions are closed by composition

Proof: Let $T_{1}$ and $T_{2}$ be two unbounded path transductions. Our aim is to show that the relation $T=T_{1} \cdot T_{2}$ remains an unbounded path transduction.

Let $\mathcal{G}$ be a graph and let $\mathcal{H}=T_{2}\left(T_{1}(\mathcal{G})\right)$ hence

$$
\mathcal{H}=h_{2}^{-1}\left(g_{2}^{-1}\left(h_{1}^{-1}\left(g_{1}^{-1}(\mathcal{G}) \times \mathcal{F}_{1}\right)\right) \times \mathcal{F}_{2}\right)
$$

where $g_{i}$ and $h_{i}$ are rational substitutions and $\mathcal{F}_{i}$ are finite graphs.

By composition of rational substitutions (Lemma 1) we deduce a rational substitution $g_{3}=g_{2} \cdot h_{1}$ such that

$$
\mathcal{H}=h_{2}^{-1}\left(g_{3}^{-1}\left(g_{1}^{-1}(\mathcal{G}) \times \mathcal{F}_{1}\right) \times \mathcal{F}_{2}\right)
$$

From Lemma 5 we deduce a rational substitution $k$, a morphism $f$ and a finite graph $\mathcal{K}$ such that

$$
g_{3}^{-1}\left(g_{1}^{-1}(\mathcal{G}) \times \mathcal{F}_{1}\right) \times \mathcal{F}_{2} \approx k^{-1}\left(f^{-1}\left(g_{1}^{-1}(\mathcal{G}) \times \mathcal{F}_{1}\right) \times \mathcal{K}\right)
$$

The function $f$ being a morphism, by Lemma 2 we get

$$
\left.g_{3}^{-1}\left(g_{1}^{-1}(\mathcal{G}) \times \mathcal{F}_{1}\right) \times \mathcal{F}_{2} \approx k^{-1}\left(f^{-1}\left(g_{1}^{-1}(\mathcal{G})\right) \times f^{-1}\left(\mathcal{F}_{1}\right)\right) \times \mathcal{K}\right)
$$

With $g=f \cdot g_{1}, h=h_{2} \cdot k$, and $\mathcal{F}=f^{-1}\left(\mathcal{F}_{1}\right) \times \mathcal{K}$ for any graph $\mathcal{G}$ we have

$$
h_{2}^{-1}\left(g_{2}^{-1}\left(h_{1}^{-1}\left(g_{1}^{-1}(\mathcal{G}) \times \mathcal{F}_{1}\right)\right) \times \mathcal{F}_{2}\right) \approx h^{-1}\left(g^{-1}(\mathcal{G}) \times \mathcal{F}\right)
$$




\section{Conclusion}

In the theory of languages and automata, there was an asymmetry between an algebraic characterization for word languages and a "mechanical" one for their acceptors. Much work has been done to exhibit useful algebraic language transformations but the corresponding acceptors transformations remained scattered and hidden in some technical lemma.

Infinite labelled graphs/automata gives a natural framework to study the behavior of acceptors. We proposed the notion of path transduction which is a graph transformation. With this definition, the correspondence between the graph transformation and the language transformation becomes clearer than it is in [17]. The $A F G$ and full-AFG defined in [17] may be respectively defined by closure under bounded and unbounded path transductions.

We also showed that path transductions are closed by composition, generalizing the classical Elgot and Mezei theorem from words to graphs. This property gives a canonical form for graphs in principal $A F G$ like pushdown graphs [13] or prefix recognizable graphs [6].

To deepen the knowledge about graphs as language acceptors, a systematic study of language-compatible graph transformations and their application on infinite automata is required. The generalization of rational word transductions to path transductions is one step in this systematic study.

Path transductions are strictly weaker than monadic transductions, but their expressive power is important: for example the transitions graphs of Petri nets are the images of hypergrids by path transductions. Another interesting aspect that is not developed here is that, being weaker than monadic transductions, path transductions preserve also weaker decidability properties like reachability under rational control.

\section{Acknowledgement}

I would like to thank the referees (especially one) for their numberous remarks.

\section{References}

1. J.-M. Autebert and L. Boasson. Transductions rationnelles - application aux langages algébriques. Masson, Paris, 1988.

2. J. Berstel. Transductions and context-free languages. B.G. Teubner, Stuttgart, 1979 .

3. A. Blumensath and E. Grädel. Automatic structures. In Proceedings of 15th IEEE Symposium on Logic in Computer Science LICS 2000, pages 51-62, 2000.

4. L. Boasson and M. Nivat. Sur diverses familles de langages fermes par transductions rationelle. Acta Informatica, 2:180-188, 1973.

5. D. Caucal. On infinite terms having a decidable monadic theory. In 27th MFCS, volume 2420 of $L N C S$, pages 165-176, Warsaw, 2002.

6. D. Caucal. On infinite transition graphs having a decidable monadic theory. Theoretical Computer Science, 290:79-115, 2003. 
7. B. Courcelle. Monadic-second order definable graph transductions : a survey. Theoretical Computer Science, 126:53-75, 1994.

8. B. Courcelle and I. Walukiewicz. Monadic second-order logic, graph coverings and unfoldings of transition systems. Annals of Pure and Applied Logic, 1998.

9. J. Engelfriet. Context-free graphs grammars. In G. Rozenberg and A. Salomaa, editors, Handbook of Formal Languages, volume 3, chapter 3, pages 125-213. SpringerVerlag, 1997.

10. S. Ginsburg. Algebraic and automata-theoretic properties of formal languages. North-Holland, Amsterdam, 1975.

11. S. Ginsburg and S. Greibach. Abstract families of languages. Mem. Am. Math. Soc., 87, 1969.

12. A. Mateescu and A. Salomaa. Handbook of Formal Languages, volume 1, chapter Aspects of classical language theory, pages 175-252. Springer-Verlag, 1997.

13. D. Muller and P. Schupp. The theory of ends, pushdown automata, and secondorder logic. Theoretical Computer Science, 37:51-75, 1985.

14. M.O. Rabin. Decidability of second-order theories and automata on infinite trees. Trans. Amer. Math. soc., 141:1-35, 1969.

15. J.-C. Raoult. A survey of tree transductions. In M. Nivat and A. Podelski, editors, Tree Automata and Languages, pages 311-325. North-Holland, Amsterdam, 1992.

16. W. Thomas. A short introduction to infinite automata. In Proceedings of the 5th international conference Developments in Language Theory, volume 2295, pages 130-144. LNCS, 2001.

17. T. Urvoy. Abstract families of graphs. In M. Ito and M. Toyama, editors, Proceedings of the 6th international conference Developments in Language Theory, volume 2450 of $L N C S$, pages 381-392, 2002.

18. T. Urvoy. Familles abstraites de graphes. PhD thesis, Université de Rennes I, 2003. 